\title{
The wide spectrum of sport and exercise medicine: an Australasian College of Sports
Physicians theme issue
} Andrew P Garnham

Turn off the TV! Too often this is the advice parents give to their children simply because they feel children should be doing other things, or the noise is annoying. Are there good reasons to turn it off? And what about adults-surely some relaxation is good? For some time we have known just how dangerous sitting down in front of the television can be in terms of cardiovascular mortality. Indeed, simply sitting in front of the television, even if it is turned off, is risky. Now we have the figures to tell us just what those risks are. ${ }^{1}$

Scarily, half an hour in front of the box once over the age of 25 may shorten life by just as much as a cigarette. Typical viewing behaviours of several hours per day can reduce life expectancy by years. The question remains, what is the really dangerous content? Does watching sport, particularly a nail-biting contest, reduce or increase the risk?

Having taken on board the straightforward and potent message that television is dangerous, we then should consider alternative activities. For the children, desirable options may include playing sport or a musical instrument. Existing dogma is that that these activities will improve coordination. However, this assumption has been critically explored to produce the surprising finding that children engaging in these activities are no better at motor imagery tasks ('coordination') than those who do not. ${ }^{2}$ Reassuringly, children rated by their parents as being clumsy are not slower, but just a little less accurate in performing coordination tasks. The takehome message is that children aged under 10 years are still developing the proprioceptive and motor planning parts of their brain-something that will come as a relief to those coaching children and frustrated at what seems like slow skill acquisition.

Balance and technique training must be a sure way of helping to protect the knee joint during a complex motor skill such as

\footnotetext{
Correspondence to Dr Andrew P Garnham, School of Exercise and Nutrition Sciences Deakin University, 221 Burwood Hwy, Burwood, New South Wales, 3125 Australia;

andrew.garnham@deakin.edu.au
}

playing Australian football. Yet another surprise awaits. Training drills specifically devised to improve knee joint biomechanics failed to effect the expected change. Sham drills were just as effective. ${ }^{3}$ Should we then simply drop these training drills? Sidestepping is an essential element of many team sports and a range of training protocols should be employed to better assess and achieve improvements in injury risk.

Research is sometimes discouraged on the basis that the possibility of a negative result means that it may not be published and so is a waste of time. To complete the triad of studies which have failed to support their hypotheses, Barwood and colleagues found that feeling cooler during high-intensity exercise did not change pacing strategies or performance outcomes. However, the negative result led on to the exploration of what is it that tells us how hard to work in challenging conditions. It appears that perceived exertion remains the most important factor but the question is whether it is a cause or consequence. A study with a negative outcome has the potential to more clearly set out the direction for future research. Knowing what does not work can be just as important as knowing what does.

New research may surprise us in its findings, but we can always rely on time-tested strategies to achieve effective treatment of common injuries. Or so we would believe. Always one to question the established paradigms, Hamilton has looked at the history of the hammy. ${ }^{4}$ Over the last 100 years, there have been many twists and turns in our hamstring management strategies and the same mistakes have been made more than once. This of course assumes that the modern-day science is right, and reminds us that sometimes the best way of looking into the future is to look into the past. Bruce Hamilton suggests that despite some setbacks, we have made progress in treatment as well as prevention.

Sexual harassment in sport is likely much more pervasive than is recognised. Most clinicians who have worked with teams over any period of time have probably experienced instances of this, but may not always have realised it. The psychological and physical consequences of sexual harassment and abuse are potentially diverse and may manifest as seemingly unrelated problems such as excessive training behaviours and unexplained fatigue. Identifying the problem is very challenging for practitioner and patient and taking the next step of dealing with it may be even more so. This study outlines the ghastly deliberate 'grooming' of an athlete that a perpetrator may use to coerce the athlete into sexual interaction. Understanding this study may help to prevent episodes such as those chronicled over a 15-year period at the Pennsylvania State University football programme (http://en.wikipedia.org/wiki/ Penn_State_child_sex_abuse_scandal). Team medical staffs have important obligations and the practical guidelines published in this issue of $B J S M$ are invaluable to help us fulfil these obligations. ${ }^{5}$

Finally, this 2012 issue of BJSM comes to Australasian College of Sports Physician (ACSP) members at our 27th Annual Scientific Conference, in Coolum, Queensland. This coincides with the second birthday of Sport and Exercise Medicine as a specialist discipline in Australia. Despite this important step, achieving real recognition of the importance of physical activity within the health and political systems remains as much a challenge in Australia as it is in many other countries. At this academic meeting we will advance the strategic vision of the ACSP, refine our education and training programmes, and share success stories relating to practice and policy influence. We will provide $B J S M$ readers with an early invitation to the 2013 ACSP Annual Scientific Conference via BJSM's blog, Twitter (@BJSM_BMJ) and Facebook channels.

Competing interests Andrew Garnham is past President of the Australasian College of Sports Physicians.

Provenance and peer review Not commissioned; internally peer reviewed.

Accepted 14 August 2012

Br J Sports Med 2012;46:895

doi:10.1136/bjsports-2012-091687

\section{REFERENCES}

1. Veerman JL, Healy GN, Cobiac LJ, et al. Television viewing time and reduced life expectancy: a life table analysis. Br J Sports Med 2012;46:927-30.

2. Dey A, Barnsley N, Mohan R, et al. Are children who play sport or a musical instrument better at motor imagery than children who do not? $\mathrm{Br} J$ Sports Med 2012;46:923-6.

3. Donnelly CJ, Elliott BC, Doyle TLA, et al. Changes in knee joint biomechanics following balance and technique training and a season of Australian football. Br J Sports Med 2012;46:917-22.

4. Hamilton B. Hamstring muscle strain injuries: what can we learn from history? Br J Sports Med 2012;46:900-3

5. Marks S, Mountjoy M, Marcus M. Sexual harassment and abuse in sport: the role of the team doctor. Br J Sports Med 2012;46:905-8. 